\title{
INVESTIGATION ÓF MISFIT DISLOCATION SOURCES IN GaAs EPITAXIAL LAYERS
}

\author{
W. Wierzchowski, K. Mazur, We. Strupiński \\ Institute of Electronic Materials Technology, Wólczyńska 133, 01-919 Warszawa, Poland
}

\section{K. WIETESKA}

Institute of Atomic Energy, 05-400 Otwock-Świerk, Poland

\section{AND W. GraefF}

DESY HASYLAB, Notkestr. 85, 22603 Hamburg, Germany

The formation of misfit dislocation was studied in GaAs homoepitaxial layers on the substrates containing considerable amount of isoelectronic indium. The layers were grown with metal-oxide chemical vapour deposition and chemical vapour deposition methods including low temperature process with tertiarbutylarsine arsenic source. The critical conditions of misfit dislocation formation were exceeded up to $5 \times$. The samples were examined before and after epitaxial process with a number of different X-ray topographic and diffractometric methods, including high resolution synchrotron white beam topography. The crystallographic identification of the defects was supported by the numerical simulation of topographic images. It was found that a number of threading dislocations, continuing in the epitaxial layer from those existing in the substrate, did not take part in the formation of misfit dislocations despite a suitable slip system. On the other hand, the formation of misfit dislocations from small imperfections of epitaxial deposit was proved in many cases. A reasonable good quality of the layers was confirmed by the resolution of individual defects and only small broadening of rocking curves.

PACS numbers: $61.10 .-\mathbf{i}$

\section{Introduction}

The very important problem in epitaxial technology is to what extent the perfection of deposit is determined by the perfection of substrate. This concerns in particular the formation of misfit dislocations, which are one of the most popular defects causing the degradation of electrical properties.

In the present paper the dependence of misfit dislocation formation on the defects existing in the substrate and those introduced to the layer is followed in 
the case of GaAs by successive use of X-ray topographic methods before and after epitaxial process. Part of the topographic investigations was performed with the use of synchrotron source DORIS III at DESY. The idea of successive examination was first realized in the case of silicon epitaxy and planar transition technology [1]. Some recent papers reported in situ observations of MBE epitaxial growth of quarternary compounds using synchrotron equipment with TV camera $[2,3]$.

The important point of the present investigation was providing conditions for effective application of X-ray topography. For this reason the thickness of epitaxial deposit was chosen in the range $3-5 \mu \mathrm{m}$. It was large enough to provide sufficient sensitivity of topographic methods and still not to cause multiplication of the defects. The substrates were cut from highly indium doped GaAs crystals with reduced dislocation density. Both metal-oxide chemical vapour deposition (MOCVD) and chemical vapour deposition (CVD) grown layers were examined.

The topographic investigation was supported by numerical simulation of the defect images. Some former results of these simulations were published elsewhere $[4,5]$.

\section{Sample preparation}

The MOCVD epitaxial growth was realized using AIXTRON AIX-200-RD apparatus. The use of tertiarbutylarsine (TBA) as arsenic source enabled decreasing in the growth temperature to $590^{\circ} \mathrm{C}$, while more common arsine requires the temperature $100^{\circ} \mathrm{C}$ larger [1]. The second component - gallium is provided as the vapours of threemethylgallium (TMGa). The characteristic feature of the MOCVD method is a slow growth-rate of $0.03 \mu \mathrm{m} / \mathrm{min}$ destined for preparation of thin multi-layer structures. The growth of $3-5 \mu \mathrm{m}$ layers required time of approximately 3 hours.

The CVD-growth was performed using older AMG-500 equipment. The method uses gallium vapour transported in the stream of $\mathrm{HCl}$. The growth is performed with ten times greater rate than in the case of MOCVD method i.e. $0.2-0.3 \mu \mathrm{m} / \mathrm{min}$ at the temperature $790^{\circ} \mathrm{C}$.

The most important results of the present work were obtained in epitaxial layers deposited on substrates containing $0.2-1 \%$ of isoelectronic indium cut from crystals grown with Czochralski and horizontal gradient freeze (HGF) methods doped simultaneously with similar concentration of indium and $10^{18} \mathrm{at} / \mathrm{cm}^{3}$ of silicon.

The role of indium in the presently used substrates consists both in the decrease in dislocation density [6] and in the introducing of change in the lattice parameter, responsible for the formation of misfit dislocations [7].

\section{X-ray topographic investigations}

Most of the investigation, especially in the case of substrates, was performed using Lang transmission and the double-crystal back-reflection topography. The Lang topographs were taken in symmetrical 220- and 400-type reflections in $\mathrm{Cu} K_{\alpha_{1}}$ and Mo $K_{\alpha_{1}}$ radiation. The double-crystal topographic method was realized in $511_{\mathrm{Ge}},-511_{\mathrm{Ge}}$ arrangement with $\mathrm{Cu} K_{\alpha_{1}}$ radiation, which was also used for examination of rocking curves and lattice parameter measurements. 
In the case of wafers with epitaxial deposit the double-crystal method can be very helpful in distinguishing defects from the layer and the top regions of the substrate. It also offers the possibility of more efficient defect identification based on the numerical simulation $[3,4]$. In view of curvature of wafers with epitaxial deposit a series of topographs with angular setting altered by a small step were usually exposed.

The samples with epitaxial deposit were also studied with the white beam synchrotron topography realized at DORIS III synchrotron source. The aim of the synchrotron experiments was to achieve better spacial resolution close to $2-5 \mu \mathrm{m}$, and to obtain sets of topographs for identification of dislocations in a relatively short time. The white beam topographs were taken, in equivalent 220- and 400-type reflections selecting low absorbing radiation $1.26 \AA$, similarly as in [8]. As single synchrotron topographs covered the strip $4 \times 20 \mathrm{~mm}^{2}$, limited by the vertical divergence of the synchrotron beam, the examination of large area was realized taking a series of slightly overlapping topographs from different regions.

\section{Results and discussion}

It was found from the topographic investigation $[5,9]$ that the substrates cut from the Czochralski-grown crystal still contain a considerable dislocation density in the outer part of the crystal, while it is lowered in the middle part of the crystals under $10^{3} / \mathrm{cm}^{2}$. The dislocations form a system of glide bands. In the case of HGF-grown crystals the density of dislocations is much lower in the whole area, but they are also grouped in some glide bands. The characteristic feature are also the striation fringes connected with lattice parameter change caused by segregation of indium dopant. The present examination included the measurements of lattice parameter change based on double-crystal measurements in $400_{\mathrm{Ge}}, \mp 400_{\mathrm{GaAs}}$ arrangements, similar to those described by Godwod et al. [10]. It was found that the measured lattice parameter in the range $5.66-5.68 \AA$ corresponds to the indium concentration $0.1-1 \%$. The upper value occurred in the end parts of the crystals. The samples cut from such regions both of Czochralski- and HGF-grown crystals usually contained a characteristic "envelope patterns" caused by cellular growth connected with growth-surface instabilities $[11,12]$.

The Lang topograph of the representative epitaxial layer deposited on the substrate cut from indium- and silicon-doped HGF-grown GaAs crystal with an array of created misfit dislocations can be found in Fig. 1. Due to the extinction the topegraph reveals only a part of dislocations present in the substrate.

The details of the misfit dislocation structure are much better seen in the case of synchrotron topographs, reproduced in larger magnification in Fig. 2. Here we may notice the examples of misfit dislocations formed from the dislocations existing in the substrates - marked with filled arrows and the dislocation which cannot be connected with the dislocations existing previously in the substrate marked with empty arrows. We may also distinguish the glide band dislocation systems which did not form misfit dislocations - marked with dotted arrows.

On the base of contrast changes and the determination of dislocation direction it was found that even the dislocations with a suitable glide system postulated by Matthews theory [7] did not produce misfit dislocation segment. This was also 


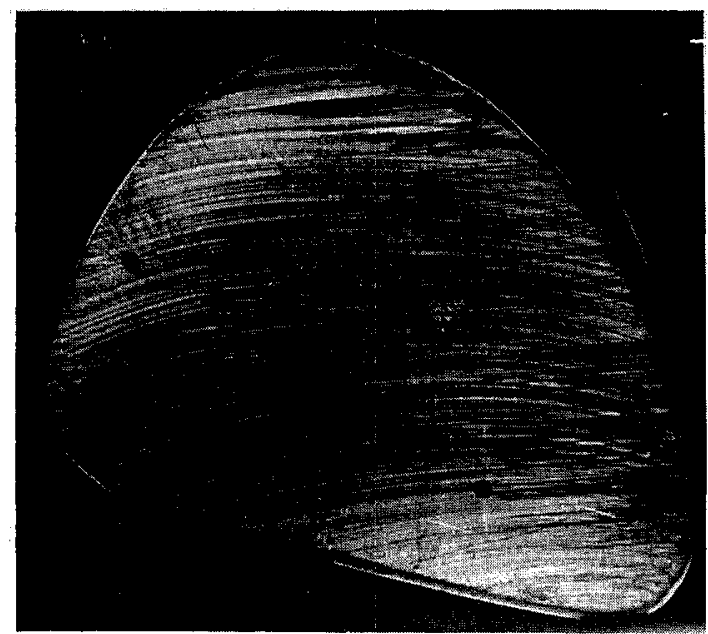

- Fig. 1. The 220 Lang topograph of the representative $4 \mu \mathrm{m}$ MOCVD grown epitaxial layer deposited on the substrate cut from HGF-grown indium and silicon doped substrate.

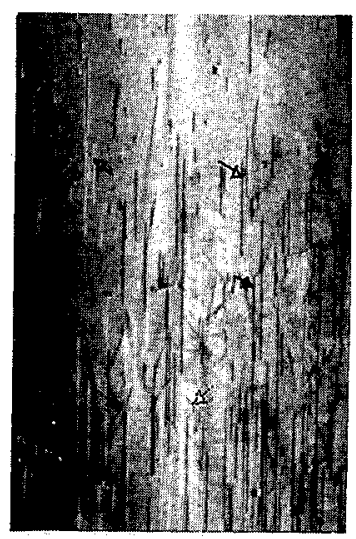

Fig. 2. $25 \times$ magnification of the white beam synchrotron projection topograph in 220 reflection selecting low absorbing $1.26 \AA$ radiation of a sample similar to shown in Fig. 1. The diffraction vector is located horizontally. Filled, empty and dotted arrows denote respectively the representative misfit dislocations created from the substrate dislocations, misfit dislocations created from the defects introduced into the layer and the threading dislocations, which did not produce the misfit dislocation segment.

confirmed using double-crystal topography supported by numerical simulation of contrast as is shown in Fig. 3. The simulated image well corresponding to the experimental image of threading dislocation was obtained assuming the same (111) inclined slip plane, which was confirmed for many misfit dislocations. In the last case the numerical simulation was also used. 

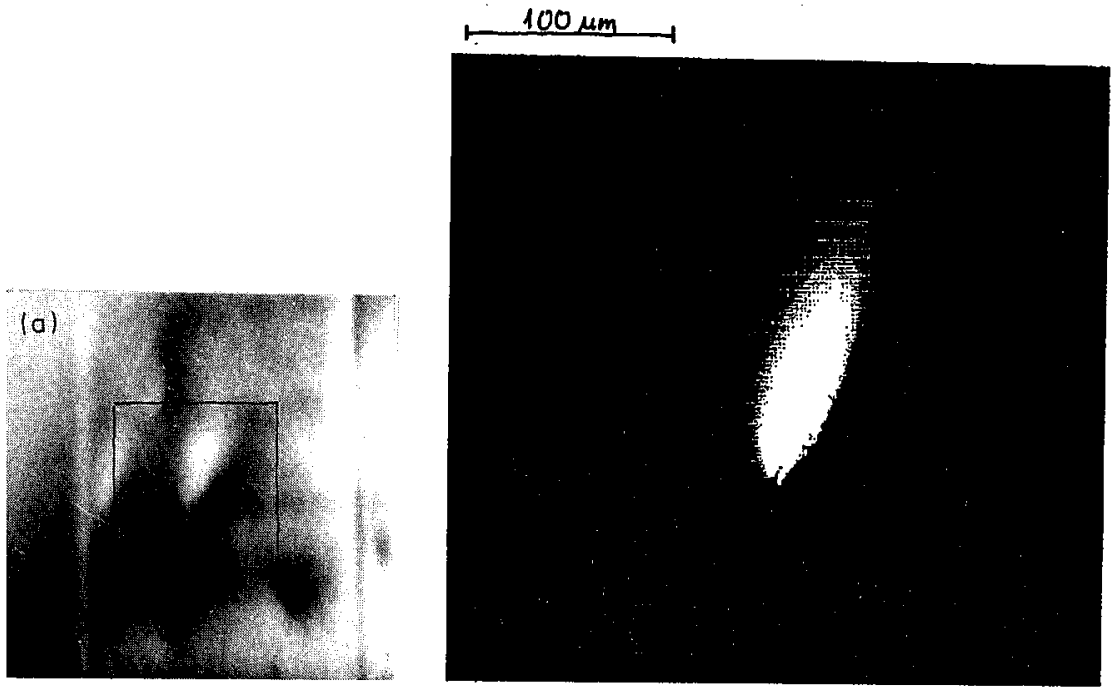

Fig. 3. Double-crystal images of threading dislocations identified as $30^{\circ}$ with Burgers vector and (111) glide plane inclined to the surface for the position at low angle side of the layer peak: (a) experimental, (b) simulated.
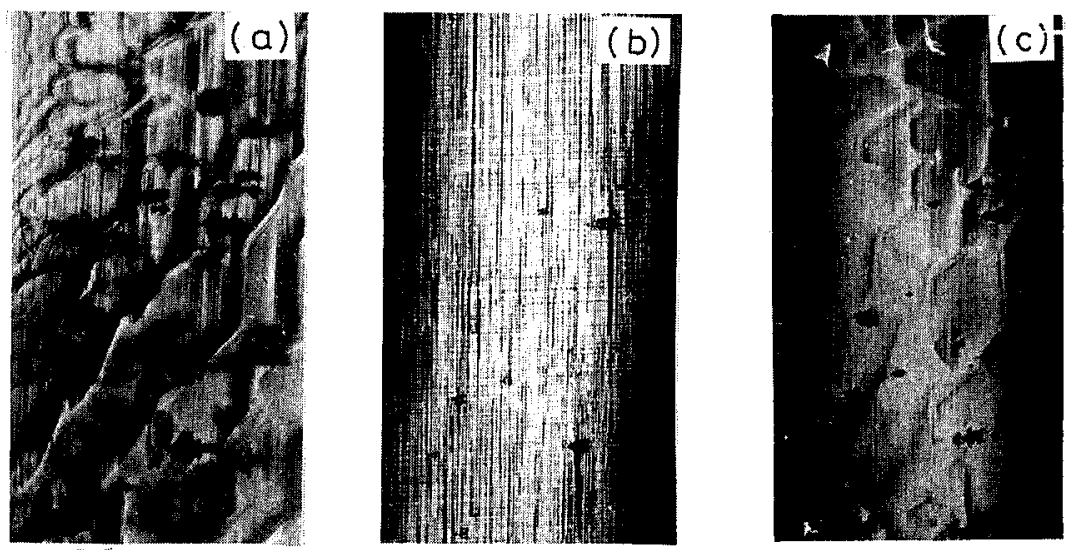

Fig. 4. The formation of misfit dislocations in the MOCVD $5 \mu \mathrm{m}$ epitaxial layer grown on the substrate cut from HGF-grown crystal with "envelope-pattern" defects due to cellular-growth (a) $220 \mathrm{Cu} K_{\alpha_{1}}$ Lang topograph of the substrate before the deposition of the layer; (b) $511,-511 \mathrm{Cu} K_{\alpha_{1}}$ double-crystal topograph of the wafer with epitaxial deposit taken at the low angle slope of the layer peak; (c) $511,-511 \mathrm{Cu} K_{\alpha_{1}}$ double-crystal topograph of the wafer with epitaxial deposit taken at the low angle slope of the substrate peak.

The reason of the above phenomena may be connected with the decoration of dislocation by the impurities, in particular by the diffusion of dopant along the 
dislocation line, or by some larger precipitations or point defect clusters stopping the glide motion of the dislocation.

At present crystal thickness we did not find a significant difference in the lattice perfection and the formation of misfit dislocation for the different methods of epitaxial growth. Usually a greater concentration of misfit dislocations was observed in the case of greater concentration of indium. The last fact also proves that the most of dislocations originated in the layer and have no connection with the defects in the substrate.

It is also illustrated by Fig. 4 showing the generation of the misfit dislocation line in the case of "envelope pattern" defects in HGF-grown crystals. The dislocation lines were not distinguishable in the substrates and a dense misfit dislocation lines array was observed. It should be noted that at the layer peak we do not see the "envelope pattern" but some local bending is present due to the misfit dislocation bundles. The rocking curves of the presently investigated samples exhibited two separate maxima only slightly broadened by the value of $2^{\prime \prime}$ with respect to the theoretical values, proving a good perfection of the layers.

\section{Conclusions}

In the present studies of MOCVD- and CVD-grown gallium arsenide epitaxial layer at slight exceeding of the critical conditions we observed a moderate density of misfit dislocations, partly formed from the dislocation continuing from the substrate and partly generated from the defects in the layer. It was found that a number of threading dislocation did not form misfit dislocation despite a suitable glide system, most probably due to the decoration with the impurities.

A good crystallographic perfection of the layer was confirmed.

The work was sponsored by the Committee for Scientific Research, grant no. $1130 / 55$.

\section{References}

[1] G.H. Schwuttke, J.H. Queisser, J. Appl. Phys. 33, 1540 (1962).

[2] W. Spirkl, B.K. Tanner, C. Whitehouse, S.J. Barnett, A.G. Cullis, A.D. Johnson, A. Keir, B. Usher, G.F. Clark, W. Hagston, C.R. Hogg, B. Lunn, Philos. Mag. A 69, 221 (1994).

[3] S.J. Barnett, C.R. Whitehouse, A.M. Keir, G.F. Clark, B. Usher, B.K. Banner, M.T. Emeny, A.D. Johnson, J. Phys. D 26, A45 (1993).

[4] W. Wierzchowski, K. Mazur, K. Wieteska, J. Phys. D 28, A33 (1995).

[5] W. Wierzchowski, Prace ITME 44 (1994) (in Polish).

[6] A. Chabli, E. Molva, A. George, F. Bertin, B. Bunod, J. Bletry, in: Proc. Symp. E-MRS, June 1986, Strasbourg, Eds. G. Bastard, H. Otpholzer, Vol. 21, p. 27.

[7] J.W. Matthews, S. Mader, T. Light, J. Appl. Phys. 41, 3800 (1970).

[8] P. Riglet, M. Sauvage, J.-F. Petroff, Y. Epelboin, Philos. Mag. A 42, 339 (1980).

[9] W. Wierzchowski, K. Mazur, Acta Phys. Pol. A 84, 789 (1993).

[10] K. Godwod, R. Kowalczyk, Z. Szmid, Phys. Status Solidi A 21, 227 (1974).

[11] B. Schumann, Krist. Techn. 11, 41 (1976).

[12] F. Minari, B. Billia, J. Cryst. Growth 140, 264 (1994). 